\title{
EFECTO DE LA PODA EN EL TRASPLANTE DEL ALMÁCIGO DE PEJIBAYE (Bactris gasipaes Kunth) ${ }^{1}$
}

\author{
Carlos Arroyo ${ }^{2}$, Jorge Mora ${ }^{3}$
}

\begin{abstract}
RESUMEN
Efecto de la poda en el trasplante del almácigo de pejibaye (Bactris gasipaes Kunth). Cinco tratamientos con diferentes combinaciones de poda de follaje y raíz en pejibaye se llevaron a cabo, al trasplantarlas al campo con raíz desnuda, y su comportamiento fue evaluado con base en siete variables. Además se evaluaron comparativamente las plántulas que mostraban la hoja emergente u hoja guía contra aquéllas en que ésta aún no era visible externamente. Las plántulas que no sufrieron poda alguna fueron significativamente superiores a todos los tratamientos con alguna forma de poda. Esto, no sólo en porcentaje de sobrevivencia $(90,33 \%)$, sino también en las otras variables estudiadas y en especial en cuanto a su desarrollo posterior, medido por el peso total de la planta tomado seis meses más tarde. Las plántulas sin follaje y sin raíz, mostraron un porcentaje de sobrevivencia del $(66,33 \%)$, y su recuperación, medida por peso de raíz y peso aéreo, seis meses más tarde, fue muy inferior a todos los tratamientos. En todos los casos, incluyendo el testigo, la presencia visible de la nueva hoja emergente u hoja guía significó un mayor porcentaje de sobrevivencia. Las siete variables utilizadas resultaron altamente correlacionados, siendo el peso total de la planta quizás el mejor evaluador, pero en el campo, el diámetro del tallo resulta más fácil de manejar en la práctica.
\end{abstract}

\begin{abstract}
Pruning effect on peach palm (Bactris gasipaes Kunth) transplanting. Five treatments combining different pruning practices of foliage and roots were conducted. Their behaviour was evaluated six months after transplanting to the field, based on seven different parameters. All were transplanted with naked roots. A comparative evaluation was done based on the external visual presence or absence of the new emerging leaf or guide leaf. The results were as follows: the plants that were not pruned, were statiscally superior to all pruning treatments tried, not only in survival percentage $(90.33 \%)$ but also in the other six variables studied, mainly in regard to the development of the plant measured by the total weight recorded six months after the transplanting. The plants with no foliage and no roots showed $63.33 \%$ of survival and their posterior recovery, as measured by the weight of the roots and aerial part of the plant, was markedly inferior to all other treatments. In all cases, including the control plots, the presence of the new emerging leaf meant a 2 to $4 \%$ increase in the survival of the plants. The seven variables used were highly correlated: plant diameter and height, number of leaves, root system and aerial part weight, total plant weight, and survival percentage.
\end{abstract}

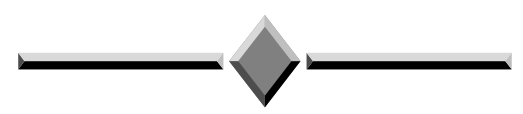

\section{INTRODUCCIÓN}

Dos aspectos se tratan de dilucidar en los experimentos con diversos tratamientos en el manejo de los almacigales. Uno de orden puramente práctico, en donde se trata de encontrar el método más eficiente y económico de producir y trasplantar las plántulas. Otro, de interés más académico, que trata de estudiar el compor- tamiento de la plántula tratada de diferentes maneras para ampliar el conocimiento teórico de la misma. Esas experiencias generalmente responden a diferentes circunstancias y exigen diversas aproximaciones al estudio. Por ejemplo, el hecho de que el trasplante del almácigo se realice en la misma finca en donde se produjo o que haya que trasladarlo a larga distancia, envuelve, además del problema de deshidratación, el factor

\footnotetext{
1 Recibido para publicación en marzo del 2002. Trabajo financiado por FITTACORI

2 Escuela de Zootecnia, Universidad de Costa Rica. E-mail: carroyo@ cariari.ucr.ac.cr. Tel: 207-5504. Fax: $224-5527$.

3 Escuela de Biología, Universidad de Costa Rica.
} 
económico del transporte y con ello un planteamiento de manejo que podría ser diferente en ambos casos.

Cyrus y Mora (1983, sin publicar) determinaron que la edad de trasplante de plántulas de pejibaye a los nueve meses fue superior a aquella de cuatro meses en cuanto a porcentaje de prendimiento o sobrevivencia. Esto significa que la deshidratación afecta las plántulas menos drásticamente cuando su desarrollo es mayor. Asimismo, encontraron que los almacigales hechos en bolsas de plástico y por lo tanto trasplantados con adobe o pilón de tierra, con follaje y antitranspirante se comportaban mejor, como era lo esperado, en contraste con plántulas a raíz desnuda o con diferentes formas de poda. Así como también, que las plántulas trasplantadas con follaje pero con poda de raíces, es más deficiente que aquéllas a la cual se le podan ambas, raíz y follaje. Esto parece explicable porque en ese primer caso la planta continua transpirando pero no puede restituir esa pérdida de agua, por la ausencia de raíces, en tanto que en el segundo caso se reduce drásticamente la transpiración. También obtuvieron un porcentaje de sobrevivencia aceptable (81\%) en plántulas a raíz desnuda, con follaje y antitranspirante, sembradas dos días después de su remoción de suelo, siempre que permanezcan cubiertas y a la sombra durante ese período. Arroyo et al. (2001) hacen énfasis en el hecho de que las plántulas con mayor diámetro del tallo soportan mejor el estrés del trasplante por tener mayores reservas de nutrientes. Además, es un hecho bien conocido que en aquellos casos en que el estrés inducido por el trasplante es drástico y produce la muerte del follaje, las plántulas con tallos de mayor diámetro resurgen, aún cuando ya parecían muertas, debido a la mayor cantidad de reservas contenidas en su "araña". Arroyo y Mora (1999), muestran además otro método de manejo de las plántulas utilizable bajo condiciones agronómicas en donde el control de la maleza es eficiente, criadas en bandejas de plástico con numerosas cavidades y trasplantadas con su pequeño adobe, con excelente sobrevivencia y desarrollo posterior.

El presente experimento se realizó con el objetivo de complementar algunos aspectos de los estudios anteriores, introduciendo otras variables en la evaluación del comportamiento de las plántulas de almácigo al momento del trasplante.

\section{MATERIALES Y MÉTODOS}

\section{Área experimental}

El ensayo se realizó en el área experimental de pejibaye que mantiene la Universidad de Costa Rica bajo el convenio MAG-UCR, en la Estación Experimental Los Diamantes, ubicada en Guápiles, cantón Pococí, provincia de Limón. La posición geográfica es $10^{\circ}$, $13^{\prime}$ latitud norte y $86^{\circ}, 46^{\prime}$ longitud oeste, y la altura es de $245 \mathrm{msnm}$. La prueba se llevó a cabo entre los meses de enero de 1999 a febrero del 2000, con temperatura promedio de $25,6^{\circ} \mathrm{C}$; una mínima de $22,5^{\circ} \mathrm{C}$ en el mes de diciembre y la máxima de $27,2^{\circ} \mathrm{C}$ en el mes de setiembre; la precipitación promedio mensual fue de $398,1 \mathrm{~mm}$, con un mínimo de $128,6 \mathrm{~mm}$ el mes de febrero y un máximo de $680,1 \mathrm{~mm}$ en diciembre.

Se realizó un análisis de suelo, antes del inicio del experimento, para tener una caracterización de las propiedades químicas del suelo, el cual se muestra a continuación (Cuadro 1).

\section{Material y diseño experimental}

Se utilizaron semillas germinadas, con las primeras dos hojas recién abiertas, de la variedad sin espinas "Diamantes 10". Esta variedad se utilizó por ser la variedad comercial de mayor producción (Arroyo y Mora 2002). Se sembraron en eras a una distancia de $5 \mathrm{~cm}$ entre plantas x $10 \mathrm{~cm}$ entre hileras. Seis meses después de sembradas se sacaron y aleatoriamente se realizó la poda según fuera el tratamiento correspondiente. Finalmente, las plantas podadas se sembraron de nuevo en un diseño de bloques al azar en parcelas subdivididas, en eras o camas a una distancia de siembra de $10 \mathrm{~cm}$ entre plantas y $10 \mathrm{~cm}$ entre hileras, distribuidas según el tratamiento (con y sin hoja emergente visible) y se evaluaron seis meses después. Durante estos dos períodos no se realizó fertilización alguna.

Todos los tratamientos, incluyendo el testigo, se trasplantaron con raíz desnuda.

Se evaluaron cinco tratamientos de poda con dos variables en cada tratamiento, especificados más

Cuadro 1. Análisis químico de suelo. Estación Experimental Los Diamantes, Guápiles, Limón, Costa Rica. 2000.

\begin{tabular}{|c|c|c|c|c|c|c|c|c|c|c|c|c|c|}
\hline \multirow{2}{*}{$\begin{array}{c}\mathbf{p H} \\
\mathrm{H}_{2} \mathrm{O}\end{array}$} & \multicolumn{4}{|c|}{$\operatorname{cmol}(+) / \mathrm{L}$} & \multirow[b]{2}{*}{ CICE } & \multicolumn{7}{|c|}{$\mathrm{mg} / \mathrm{L}$} & \multirow{2}{*}{$\begin{array}{c}\% \\
\text { M.O }\end{array}$} \\
\hline & $\mathrm{Ca}$ & Mg & $\mathbf{K}$ & Acidez & & $\mathbf{P}$ & $\mathbf{C u}$ & $\mathrm{Fe}$ & Mn & Zn & B & $\mathbf{S}$ & \\
\hline 5,2 & 4,66 & 1,58 & 0,45 & 0,58 & 7,27 & 23,1 & 15,8 & 202 & 11,4 & 0,94 & 1,22 & 6,85 & 9,96 \\
\hline
\end{tabular}


adelante. Se incluyeron cinco repeticiones por tratamiento y 30 plantas por parcela, con sub-parcela de 15 plantas, para un total de 150 plantas por tratamiento.

Los tratamientos fueron los siguientes (al momento del trasplante): 1. testigo, plantas con follaje y con raíz - con y sin hoja emergente visible; 2 . con follaje y mitad del sistema radical - con y sin hoja emergente visible; 3. con follaje y sin raíz - con y sin hoja emergente visible; 4. sin follaje y mitad del sistema radical - con y sin hoja emergente visible; 5 . con poda total de follaje y sistema radical - con y sin hoja emergente visible.

Las variables a evaluar fueron las siguientes (seis meses después del trasplante): 1. diámetro del tallo; 2.altura del tallo; 3. número de hojas; 4 . peso de la raíz; 5. peso aéreo (tallo + follaje); 6. peso total de la planta (raíz + tallo + follaje); $7 . \%$ de sobrevivencia en cada tratamiento.

Los resultados se analizaron en un Paquete de Análisis Estadístico (SAS), se obtuvieron medias y desviaciones estándares de los tratamientos, regresiones y correlaciones, entre las variables; contrastes y estimados entre tratamientos con una significancia $\mathrm{P}(\alpha<0,05)$. También se analizaron las medias con el programa SAS, para realizar los gráficos y regresiones.

\section{RESULTADOS}

Los Cuadros 2 y 3 muestran una amplia superioridad del trasplante de las plántulas sin alterar, esto es con follaje y raíz (testigo) sobre todas aquellas podadas de alguna manera, en todas la variables evaluadas y fundamentalmente reflejada en los pesos del sector aéreo de la planta y de la raíz. Asímismo, muestraron superioridad en el porcentaje de sobrevivencia (Figura 1) que, adicionalmente al mayor y mejor desarrollo de la plántula, harán que estas plantas sean más precoces y requerirán menor gasto en resiembra y asistencia.

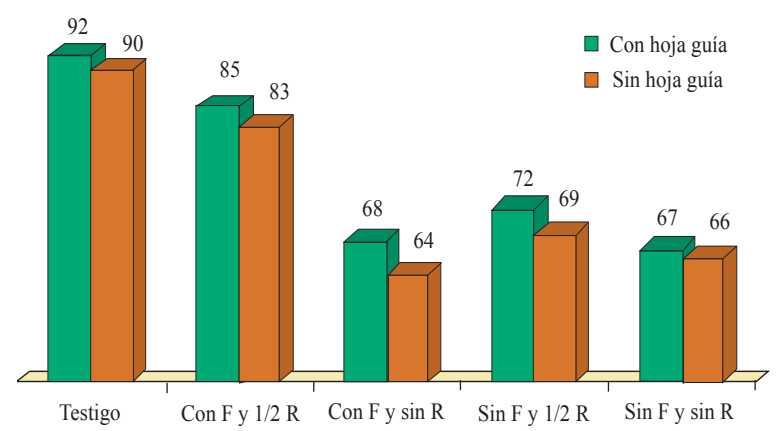

Figura 1. Sobrevivencia de plantas $(\%)$ de pejibaye en cada tratamiento con y sin hoja guía visible. Estación Experimental Los Diamantes, Guápiles, Limón, Costa Rica. 2000.

Hay aquí un factor importante adicional nuevo, mostrado tanto en los Cuadros 2 y 3 , y principalmente en el Cuadro 4, en el que las plántulas que muestran una hoja guía emergente, indiferentemente de si la plántula ha sufrido algún tipo de poda o no, se comportan significativamente mejor, respecto a peso aéreo y de la raíz, que aquéllas cuya nueva hoja guía aún no es visible. La ausencia de esa nueva hoja guía se presenta cuando la hoja guía anterior está en la fase de apertura de su lámina. Es posible que la hoja emergente, cuando ya es visible, elabore hormonas de crecimiento en mayor cantidad en este estado, cuando su propio desarrollo es más activo, favoreciendo así este comportamiento.

El Cuadro 5 muestra con claridad como los contrastes estimados entre tratamientos favorecen notoriamente las plantas no alteradas (testigo) con algún tipo de poda. También resalta la interacción positiva que existe entre raíz y follaje respecto a las variables estudiadas.

Todas las variables están correlacionadas entre sí $(\mathrm{R} 2 \geq 0,62$ a 0,92$)($ Cuadro 6). Ello indica que cualquiera de ellas constituye una característica que permite evaluar el grado en que los tratamientos de poda resultan beneficiosos o perjudiciales. Se considera el peso

Cuadro 2. Promedios utilizando plantas "sin hoja emergente visible". Seis meses después del trasplante. Estación Experimental Los Diamantes, Guápiles, Limón, Costa Rica. 2000.

\begin{tabular}{lcccrcc}
\hline $\begin{array}{c}\text { Tratamientos } \\
\text { sin hoja } \\
\text { emergente }\end{array}$ & $\begin{array}{c}\text { Diámetro planta } \\
(\mathbf{c m})\end{array}$ & $\begin{array}{c}\text { Altura tallo } \\
(\mathbf{c m})\end{array}$ & $\mathbf{N}^{\circ}$ hojas & $\begin{array}{c}\text { Peso raíz } \\
(\mathbf{g})\end{array}$ & $\begin{array}{c}\text { Peso aéreo } \\
(\mathbf{g})\end{array}$ & $\begin{array}{c}\text { Sobrevivencia } \\
(\%)\end{array}$ \\
\hline Testigo & $4,94 \mathrm{a}^{*}$ & $86,28 \mathrm{a}$ & $6,90 \mathrm{a}$ & $147,38 \mathrm{a}$ & $752,26 \mathrm{a}$ & $90,33 \mathrm{a}$ \\
Con follaje y_raíz & $3,19 \mathrm{~b}^{*}$ & $53,63 \mathrm{~b}$ & $5,24 \mathrm{~b}$ & $97,20 \mathrm{~b}$ & $253,75 \mathrm{~b}$ & $83,67 \mathrm{~b}$ \\
Con follaje y sin raíz & $1,73 \mathrm{c}^{*}$ & $27,25 \mathrm{c}$ & $2,92 \mathrm{~cd}$ & $29,21 \mathrm{c}$ & $109,58 \mathrm{c}$ & $64,67 \mathrm{c}$ \\
Sin follaje y_raíz & $1,79 \mathrm{c}$ & $27,63 \mathrm{c}$ & $3,16 \mathrm{c}$ & $35,30 \mathrm{c}$ & $101,32 \mathrm{c}$ & $69,67 \mathrm{c}$ \\
Sin follaje y sin raíz & $1,89 \mathrm{c}$ & $17,37 \mathrm{~d}^{*}$ & $2,23 \mathrm{~d}$ & $7,79 \mathrm{~d}$ & $43,55 \mathrm{~d}$ & $66,33 \mathrm{c}$ \\
\hline
\end{tabular}

*a,b,c,d, Valores con letra distinta en la misma columna son estadísticamente diferentes Duncan $(\mathrm{P} \geq 0,05)$. 
Cuadro 3. Promedios utilizando plantas "con hoja emergente visible". Seis meses después del trasplante. Estación Experimental Los Diamantes, Guápiles, Limón, Costa Rica. 2000.

\begin{tabular}{lcccccc}
\hline $\begin{array}{c}\text { Tratamientos } \\
\text { con hoja } \\
\text { emergente }\end{array}$ & $\begin{array}{c}\text { Diámetro planta } \\
(\mathbf{c m})\end{array}$ & $\begin{array}{c}\text { Altura tallo } \\
(\mathbf{c m})\end{array}$ & $\mathbf{N}^{\circ}$ hojas & $\begin{array}{c}\text { Peso raíz } \\
(\mathbf{g})\end{array}$ & $\begin{array}{c}\text { Peso aéreo } \\
(\mathbf{g})\end{array}$ & $\begin{array}{c}\text { Sobrevivencia } \\
\mathbf{\%}\end{array}$ \\
\hline Testigo & $5,35 \mathrm{a}^{*}$ & $86,85 \mathrm{a}$ & $7,17 \mathrm{a}$ & $192,88 \mathrm{a}$ & $845,06 \mathrm{a}$ & $92,33 \mathrm{a}$ \\
Con follaje y_raíz & $3,29 \mathrm{~b}^{*}$ & $57,21 \mathrm{~b}$ & $5,46 \mathrm{~b}$ & $119,64 \mathrm{~b}$ & $371,14 \mathrm{~b}$ & $85,00 \mathrm{~b}$ \\
Con follaje y sin raíz & $1,97 \mathrm{c}^{*}$ & $30,51 \mathrm{c}$ & $3,43 \mathrm{~cd}$ & $38,43 \mathrm{~cd}$ & $152,95 \mathrm{c}$ & $68,67 \mathrm{c}$ \\
Sin follaje y_raíz & $2,54 \mathrm{c}$ & $34,13 \mathrm{c}$ & $4,22 \mathrm{c}$ & $64,56 \mathrm{c}$ & $188,99 \mathrm{c}$ & $72,67 \mathrm{c}$ \\
Sin follaje y sin raíz & $1,44 \mathrm{c}$ & $23,30 \mathrm{c}$ & $2,60 \mathrm{~d}^{*}$ & $15,11 \mathrm{~d}$ & $73,57 \mathrm{~d}$ & $68,00 \mathrm{c}$ \\
\hline
\end{tabular}

*a,b,c,d, Valores con letra distinta en la misma columna son estadísticamente diferentes Duncan $(\mathrm{P} \geq 0,05)$.

Cuadro 4. Promedio de todas las variables para determinar diferencias al usar plantas con y sin hoja guía visible. Seis meses después del trasplante. Estación Experimental Los Diamantes, Guápiles, Limón, Costa Rica. 2000.

\begin{tabular}{cccccccc}
\hline \multicolumn{1}{c}{ Tratamientos } & $\begin{array}{c}\text { Diámetro planta } \\
(\mathbf{c m})\end{array}$ & $\begin{array}{c}\text { Altura tallo } \\
(\mathbf{c m})\end{array}$ & $\mathbf{N}^{\circ}$ hojas & $\begin{array}{c}\text { Peso raíz } \\
(\mathbf{g})\end{array}$ & $\begin{array}{c}\text { Peso aéreo } \\
(\mathbf{g})\end{array}$ & $\begin{array}{c}\text { Peso total } \\
(\mathbf{g})\end{array}$ & $\begin{array}{c}\text { Sobrevivencia } \\
(\%)\end{array}$ \\
\hline Plantas con hoja guía & $2,91 \mathrm{a}^{*}$ & $46,40 \mathrm{a}$ & $4,58 \mathrm{a}$ & $86,13 \mathrm{a}$ & $326,34 \mathrm{a}$ & $412,47 \mathrm{a}$ & $77,33 \mathrm{a}$ \\
Plantas sin hoja guía & $2,71 \mathrm{a}$ & $42,43 \mathrm{a}$ & $4,09 \mathrm{a}$ & $63,38 \mathrm{~b}$ & $252,09 \mathrm{~b}^{*}$ & $315,47 \mathrm{~b}$ & $74,93 \mathrm{a}$ \\
\hline
\end{tabular}

*a,b, Valores con letra distinta en la misma columna son estadísticamente diferentes Duncan $(\mathrm{P} \geq 0,05)$.

total de la planta como el más consistente, pero es el diámetro del tallo la variable más fácil de medir y resulta altamente eficiente en este sentido y en este estado de desarrollo, como también se encontró en un trabajo realizado anteriormente (Arroyo y Mora, 2002).

Las regresiones de interés teórico y práctico, ilustradas en las Figuras 2 a 11, nos indican valores que son elevados, todos ellos superiores a $\mathrm{R} 2 \geq 0,91$. De especial interés es aquel entre peso aéreo y peso de la raíz (Figura 2) por resaltar una vez más que el desarrollo del sector aéreo de la plántula correspondió a un desarrollo paralelo de la raíz. En este sentido sería importante evaluar si existen diferencias, entre variedades. Así como

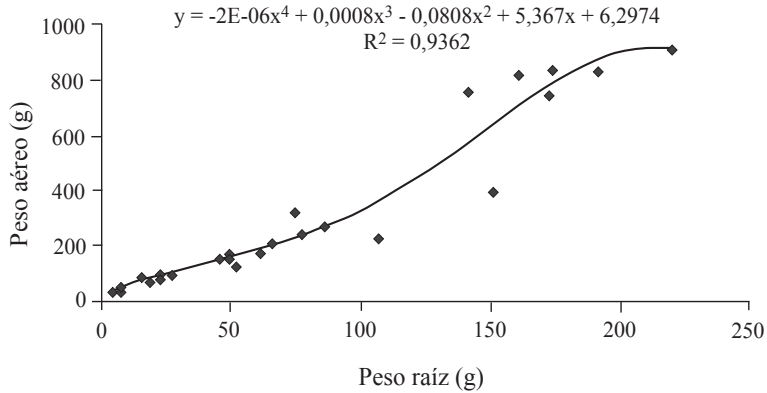

Figura 2. Regresión polinomial entre peso de la raíz y el peso aéreo,en pejibaye. Estación Experimental Los Diamantes, Guápiles, Limón, Costa Rica. 2000.

Cuadro 5. Comparaciones de contrastes estimados entre tratamientos. Estación Experimental Los Diamantes, Guápiles, Limón, Costa Rica. 2000.

\begin{tabular}{lcccccc}
\hline $\begin{array}{l}\text { Comparación entre } \\
\text { tratamientos }\end{array}$ & $\begin{array}{c}\text { Diámetro planta } \\
(\mathbf{c m})\end{array}$ & $\begin{array}{c}\text { Altura tallo } \\
(\mathbf{c m})\end{array}$ & $\begin{array}{c}\mathbf{N}^{\circ} \text { hojas } \\
\text { Peso raíz } \\
(\mathbf{g})\end{array}$ & $\begin{array}{c}\text { Peso aéreo } \\
(\mathbf{g})\end{array}$ & $\begin{array}{c}\text { Peso total } \\
(\mathbf{g})\end{array}$ \\
\hline $\begin{array}{l}\text { Testigo } v s \text { con follaje } \\
\text { y } 1 / 2 \text { raíz }\end{array}$ & 1,90 & 31,15 & 1,69 & 61,71 & 486,21 & 544,43 \\
$\begin{array}{l}\text { Testigo } v s \text { sin follaje } \\
\text { y sin raíz }\end{array}$ & 3,48 & 66,23 & 4,62 & 158,68 & 740,11 & 898,50 \\
Testigo $v s$ con follaje & & & & & & \\
y sin raíz & 3,29 & 57,69 & 3,86 & 136,31 & 667,40 & 796,51 \\
Con follaje $v s$ sin follaje & 0,63 & 16,54 & 1,21 & 40,43 & 120,00 & 171,77 \\
1/2 raíz $v s$ sin raíz & 0,94 & 18,54 & 1,72 & 56,54 & 133,89 & 182,30 \\
Interacción raíz/follaje & 0,45 & 8,00 & 0,45 & 18,06 & 47,29 & 69,80 \\
\hline
\end{tabular}


Cuadro 6. Correlaciones entre las variables. Estación Experimental Los Diamantes, Guápiles, Limón, Costa Rica. 2000.

\begin{tabular}{llllllll}
\hline & Diámetro de planta & Altura de planta & $\mathbf{N}^{\circ}$ de hojas & Peso de raíz & Peso aéreo & Peso total & Sobrevivencia \\
\hline $\begin{array}{l}\text { Diámetro } \\
\text { de planta }\end{array}$ & 1,0000 & 0,87484 & 0,86262 & 0,70904 & 0,91172 & 0,91881 & 0,76636 \\
Altura de & 0,87484 & 1,0000 & 0,75252 & 0,67712 & 0,82564 & 0,84138 & 0,75060 \\
planta & 0,0001 & & 0,0001 & 0,0001 & 0,0001 & 0,0001 & 0,0001 \\
$\mathrm{~N}^{\circ}$ de hojas & 0,86262 & 0,75252 & 1,0000 & 0,62333 & 0,75815 & 0,76284 & 0,68653 \\
& 0,0001 & 0,0001 & & 0,0001 & 0,0001 & 0,0001 & 0,0001 \\
Peso de raíz & 0,70904 & 0,67712 & 0,62333 & 1,0000 & 0,68460 & 0,79806 & 0,56746 \\
& 0,0001 & 0,0001 & 0,0001 & & 0,0001 & 0,0001 & 0,0001 \\
Peso aéreo & 0,91172 & 0,82564 & 0,75815 & 0,68460 & 1,0000 & 0,98037 & 0,73128 \\
& 0,0001 & 0,0001 & 0,0001 & 0,0001 & & 0,0001 & 0,0001 \\
Peso total & 0,91881 & 0,84138 & 0,76284 & 0,79806 & 0,98037 & 1,0000 & 0,72940 \\
& 0,0001 & 0,0001 & 0,0001 & 0,0001 & 0,0001 & & 0,0001 \\
Sobrevivencia & 0,76636 & 0,75060 & 0,68653 & 0,56746 & 0,73128 & 0,72940 & 1,0000 \\
& 0,0001 & 0,0001 & 0,0001 & 0,0001 & 0,0001 & 0,0001 & \\
\hline
\end{tabular}

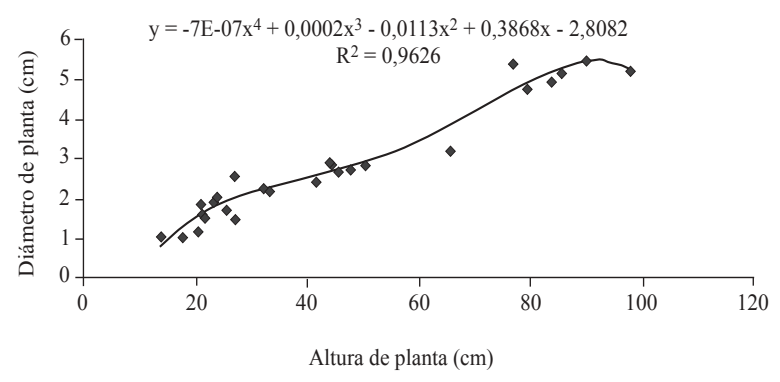

Figura 3. Regresión polinomial entre el diámetro de la planta y altura de planta, en pejibaye. Estación Experimental Los Diamantes, Guápiles, Limón, Costa Rica. 2000.

también reconfirma la estrecha relación, de interés práctico, entre el diámetro de la plántula, tanto con el peso de la raíz (Figura 5) como el peso aéreo (Figura 6) y por lo tanto con el peso total.

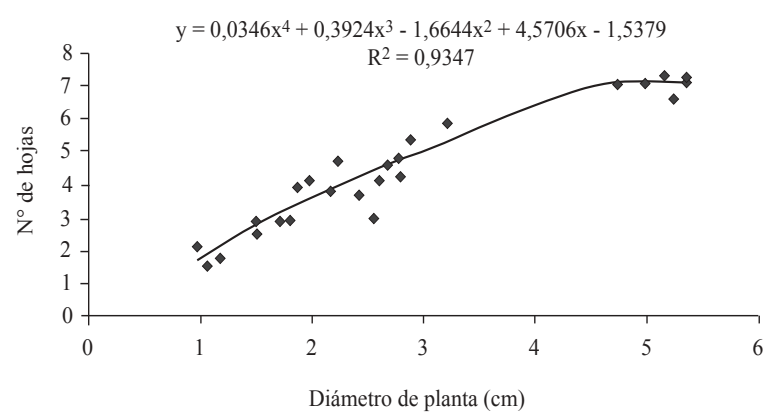

Figura 4. Regresión polinomial entre diámetro de planta y el número de hojas, en pejibaye. Estación Experimental Los Diamantes, Guápiles, Limón, Costa Rica. 2000.
Cyrus y Mora (1983), utilizando la variedad Utilis-Tucurrique obtuvieron un resultado contradictorio con el obtenido aquí respecto al tratamiento "poda de raíz, con follaje" contra "poda de raíz y follaje". En

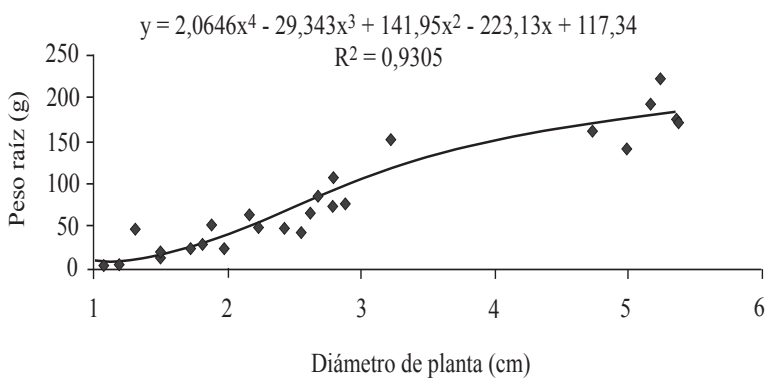

Figura 5. Regresión polinomial entre el diámetro de la planta y el peso de la raíz, en pejibaye. Estación Experimental Los Diamantes, Guápiles, Limón, Costa Rica. 2000.

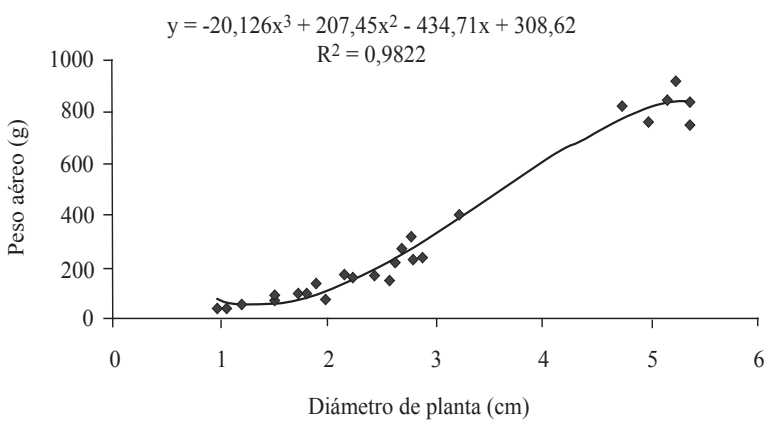

Figura 6. Regresión polinomial entre el diámetro de la planta y el peso aéreo, en pejibaye. Estación Experimental Los Diamantes, Guápiles, Limón, Costa Rica. 2000. 
el primer estudio mencionado, el último tratamiento fue superior, en tanto que aquí resultó considerablemente inferior. Esta diferencia en los resultados obtenidos podría deberse a la marcada diferencia genotípica y de comportamiento entre ambas variedades.

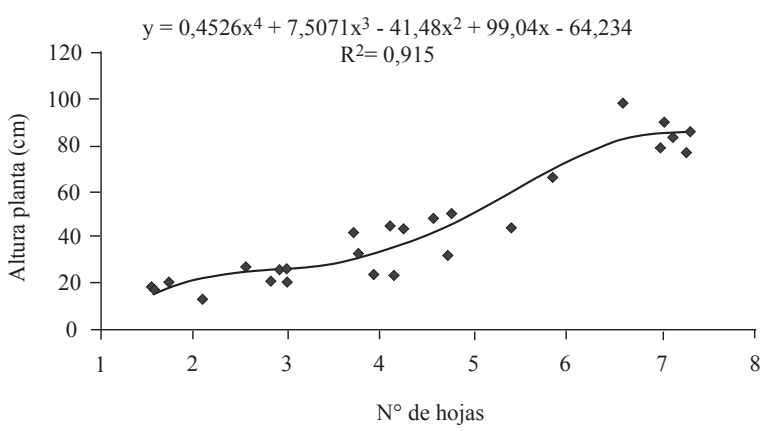

Figura 7. Regresión polinomial entre la altura de la planta y número de hojas, en pejibaye. Estación Experimental Los Diamantes, Guápiles, Limón, Costa Rica. 2000.

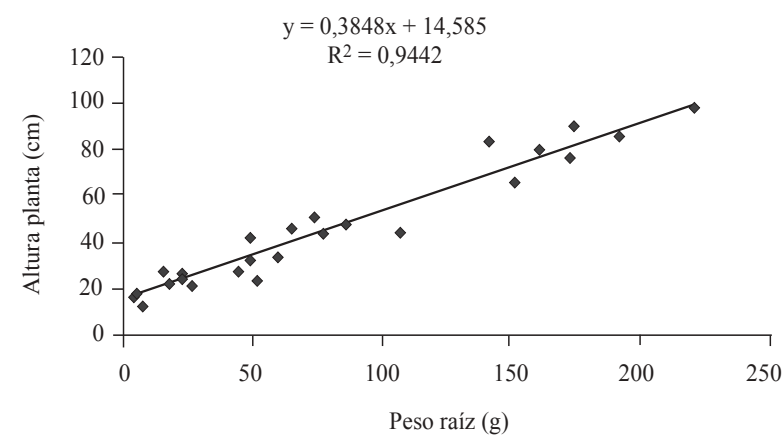

Figura 8. Regresión lineal entre la altura de la planta y el peso de la raíz, en pejibaye. Estación Experimental Los Diamantes, Guápiles, Limón, Costa Rica. 2000.

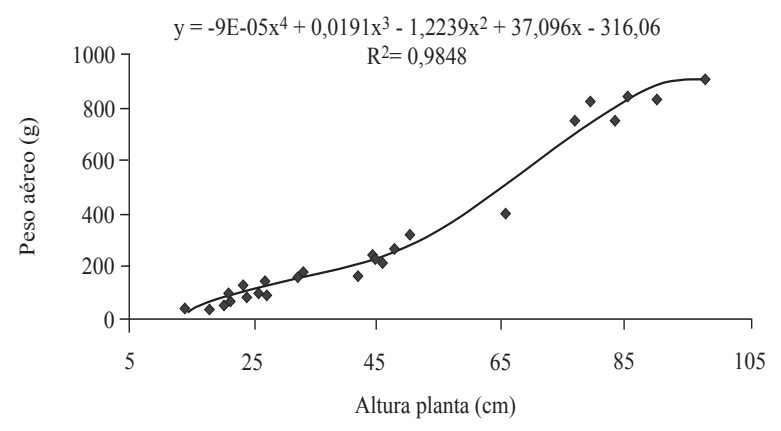

Figura 9. Regresión polinomial entre la altura de la planta y el peso aéreo, en pejibaye. Estación Experimental Los Diamantes, Guápiles, Limón, Costa Rica. 2000.

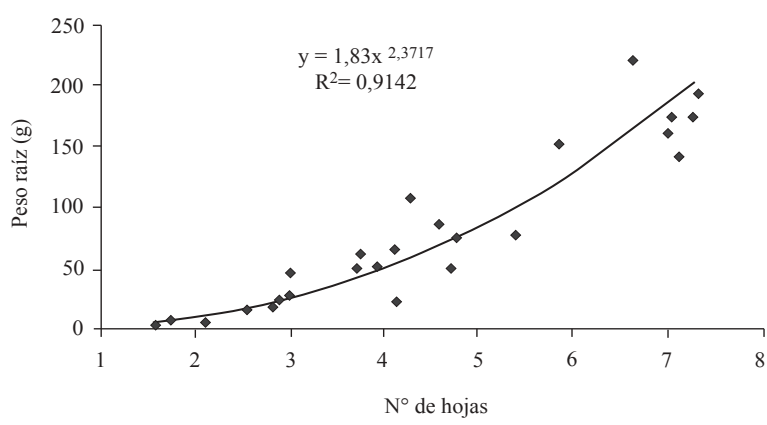

Figura 10. Regresión potencial entre la altura de la planta y el número de hojas, en pejibaye. Estación Experimental Los Diamantes, Guápiles, Limón, Costa Rica. 2000.

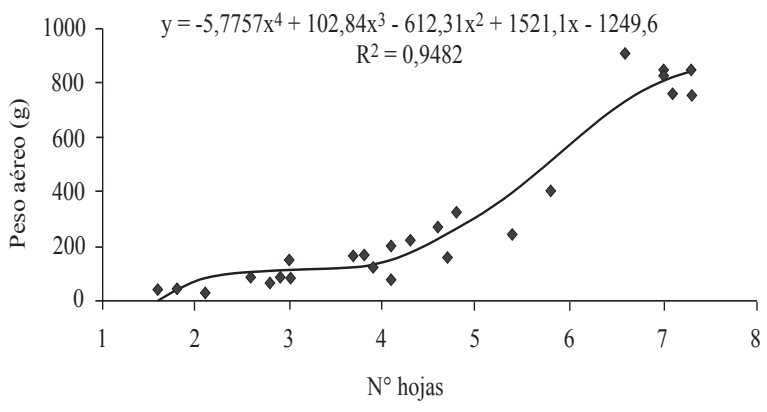

Figura 11. Regresión polinomial entre el número de hojas y el peso aéreo, en pejibaye. Estación Experimental Los Diamantes, Guápiles, Limón, Costa Rica. 2000.

\section{LITERATURA CITADA}

ARROYO, C.; MORA, J. 1999. Almácigos de pejibaye. In: Palmito de pejibaye (Bactris gasipaes Kunth) su cultivo e industrialización. Mora-Urpí, J.; Gainza, J. eds. San José, Costa Rica, Editorial Universidad de Costa Rica. pp.58-69.

ARROYO,C.; MORA, J.; SOTO, M. 2001. Fertilización de almácigos de pejibaye (Bactris gasipaes Kunth) y algunas relaciones fenológicas. Agronomía Mesoamericana 12(2): 153-159.

ARROYO,C.; MORA, J. 2002. Producción comparativa de palmito entre cuatro variedades de pejibaye (Bactris gasipaes Kunth). Agronomía Mesoamericana 13(2): 137-142.

CYRUS,E.; MORA-URPÍ. J. 1983. Métodos de trasplante en pejibaye (Bactris gasipaes). Sin publicar. 